\title{
In Bewegung bleiben oder: 20 Jahre FaDaF ${ }^{1}$
}

\author{
Klaus Vorderwülbecke
}

\section{Zusammenfassung}

Der Beitrag gibt einen Überblick über die Entwicklung und die Aufgaben des Fachverbandes Deutsch als Fremdsprache (FaDaF) seit seiner Gründung 1989/90. Er zeigt dabei die Entwicklungslinien des Verbandes auf, der als Nachfolge-Organisation des Arbeitskreises Deutsch als Fremdsprache beim DAAD (AkDaF) dessen Aufgaben übernommen, fortgeführt und weiter entwickelt hat.

\section{Liebe Kolleginnen und Kollegen!}

Dieses Gebäude und damit auch dieser Hörsaal stammen aus dem Jahr 1998. Ich vermute, es ist das erste Mal, dass hier gefüllte Sektgläser auf den Pulten stehen. Das allein wäre schon ein guter Grund gewesen, einen Fachverband $\mathrm{DaF}$ zu gründen. Sekt im Hörsaal, das wird uns hoffentlich nicht als Ausdruck spätgermanischer Dekadenz angekreidet. Ich will hier keinen Festvortrag halten. Mein Auftrag war ja nur, auf zwanzig Jahre FaDaF zurückzublicken.

Dabei wollte ich mich nicht allein auf mein schwächelndes Gedächtnis verlassen und habe Weggefährten befragt und darüber hinaus viele Informationen von Frau Middeke und Frau Schikowski in der Geschäftsstelle sowie von Matthias Jung und Armin Wolff bekommen.

Bei den ersten Nachforschungen gab es schon gleich eine Überraschung: Der $\mathrm{FaDaF}$ wurde gar nicht 1990 gegründet, sondern am 7. September 1989 (vgl. Info DaF 16/1989: 492). Wir sind also dreiviertel Jahre zu spät mit der Feier. So könnten wir eigentlich die Sektgläser wieder einsammeln.

Es gibt aber doch einen ganz guten Grund, heute den 20. Geburtstag zu feiern, weil die erste Jahrestagung und die erste Mitgliederversammlung des neuen Verbandes Anfang Juni 1990 in Bonn stattgefunden haben, wo auch der Eintrag ins Vereinsregister 1990 erfolgte. So hätten wir diesen Geburtstag also gerettet.

\section{Der AkDaF}

Der $\mathrm{FaDaF}$ hat eine längere Vorgeschichte. Die Jüngeren in diesem Raum im Jahr der Fußballweltmeisterschaft wäre das die U 40 - wissen wahrscheinlich kaum etwas über das Vorleben des FaDaF. Dieses Vorleben hatte einen Namen, und zwar »Arbeitskreis Deutsch als

1 Dies ist die schriftliche Fassung des Vortrags, den ich auf der Mitgliederversammlung der Jahrestagung 2010 des FaDaF am 14. Mai 2010 gehalten habe. Wegen zeitlicher Beschränkungen konnte ich einige Passagen nicht vortragen, die in der vorliegenden Fassung enthalten sind. Ich habe auch einige kleinere Korrekturen vorgenommen und zum besseren Verständnis einiger Zitate Anmerkungen eingefügt. Darüber hinaus wurden Literaturangaben und ein Anhang mit den Themen der Jahrestagungen und der Fachtagungen seit 1990 angefügt. 
Fremdsprache beim DAAD «, abgekürzt AkDaF, der 1973 in Erlangen gegründet wurde. $\mathrm{Zu}$ dieser Zeit war der Bereich »Deutsch für Ausländer«, wie er oft noch genannt wurde, besonders im Hochschulbereich wenig strukturiert und noch weniger fundiert und meist eine Abteilung der Auslandsämter, also Teil der Hochschulverwaltung. Und vor allem: Die Akteure hatten wenig Kontakt miteinander. Die Gründung des AkDaF war so etwas wie eine Selbsthilfegruppe, die diese Mängel überwinden wollte. Im Verständnis dieses Arbeitskreises sollte Deutsch als Fremdsprache ein Fach aus der Praxis für die Praxis sein, eingebunden in den Lehr- und Forschungsbetrieb einer Fakultät.

Der AkDaF hat dann sehr schnell eine Heimat und eine Nährmutter im DAAD gefunden. Dessen damaliger Präsident, Hansgerd Schulte, hat das gegenseitige Interesse 1974 so formuliert:

"Die Unterstützung, die der DAAD dem Arbeitskreis Deutsch als Fremdsprache [...] leistet, entspringt dem Selbstverständnis des DAAD, nicht nur Förderungsprogramme für ausgewählte Gruppen von ausländischen und deutschen Hochschulangehörigen durchzuführen, sondern auch eine Gesprächsplattform zu bieten für Fragen des internationalen Hochschulaustausches, insbesondere des Ausländer- und des Auslandsstudiums«. (Schulte in: Info DaF 1/1974: 1)

Der AkDaF musste all das z. T. in Kooperation erarbeiten, was in den Fremdsprachenphilologien weitgehend durch staatliche Kultusbehörden geleistet wurde und wird:

- Strukturen entwickeln

- Rahmenrichtlinien und Curricula erarbeiten
- Prüfungen entwickeln, vor allem die PNdS

- Materialien und Anleitungen zu diesen Prüfungen erstellen.

Was in diesen 18 Jahren geleistet wurde, kann man zumindest für den Hochschulbereich als Fachwerdung des Faches Deutsch als Fremdsprache bezeichnen.

Ein Meilenstein im Aufschwung des Faches war die Einrichtung des Lehrstuhls für Deutsch als Fremdsprache an der Ludwig-Maximilians-Universität in München 1978. Harald Weinrich, der erste Inhaber dieses Lehrstuhls, formulierte die Stimmung zu diesem Zeitpunkt wie folgt:

»[...] von einem leichten, angenehmen Rückenwind bewegt, hat sich der Forschungsund Lehrbereich Deutsch als Fremdsprache im letzten Jahrzehnt in das Gefüge der akademischen Disziplinen hineingeschoben. Nun haben wir ein neues Fach«. (Weinrich 1979: 15)

Bemerkenswert ist, dass hier schon von »Forschung « und »akademischer Disziplin« die Rede ist. Diese Jahre markieren sozusagen den Beginn der akademischen Karriere von Deutsch als Fremdsprache.

Wir sind in unserem Rückblick jetzt am Ende der 80er Jahre angelangt. Der AkDaF hatte die Pubertät schon hinter sich, und als die Volljährigkeit näher rückte, gab es stärker werdenden Druck, die Abnabelung vom DAAD zu vollziehen. ${ }^{1}$

In der Rückschau mag der AkDaF als Vorlauf oder als ein 18-jähriges Trainingslager erscheinen. In Wirklichkeit war er mehr, weil in vielen Arbeitsfeldern Strukturen und auch Publikationsformen entwickelt wurden, auf denen der FaDaF aufbauen konnte.

1 Zur Arbeit der ersten fünf Jahre vgl. Wolff 1979: 12 ff.; außerdem: Wolff/Eggers/Ehnert/ Kirsch 1997. 


\section{Die Gründung des FaDaF}

Die Gründung des FaDaF war mehr als nur eine Abnabelung, denn ein neuer Verband wurde etabliert mit allen Hoffnungen und auch Ängsten. Bei einem solchen Schritt in die formale Unabhängigkeit können zwei Prinzipien zum Tragen kommen: das Hermann-Prinzip und das Helmut-Prinzip. Das Hermann-Prinzip steht für Aufbruch, für Hoffnung, für neue Ideen, also für den Zauber, der jedem Anfang innewohnt.

Es muß das Herz bei jeder Lebensstufe Bereit zum Abschied sein und Neubeginne, Um sich in Tapferkeit und ohne Trauern In andre, neue Bindungen zu geben. (Hermann Hesse: Stufen)

Das Helmut-Prinzip ist eher pragmatisch, down to earth. Dessen Leitsatz lautet: "Entscheidend ist, was hinten rauskommt «. (Helmut Kohl, Pressekonferenz, 31.8.1984) Ich meine, dass je nach den aktuellen Notwendigkeiten bei der Entwicklung des FaDaF beide Prinzipien zu ihrem Recht gekommen sind. Hilfreich war aber vor allem, dass der DAAD seine Begleitung und finanzielle Unterstützung aufrechterhielt.

Die Gründung am 7. September 1989 also zwei Monate vor dem Fall der Mauer - war eher unspektakulär. Er geschah sozusagen in kleinem Kreis. Im Bericht über die Mitgliederversammlung 1990 in Bonn - knapp vier Monate vor der Wiedervereinigung - wird der neue Verband in einem Bericht zur 18. Jahrestagung in Bonn eher ex negativo und implizit erwähnt. Es heißt da:

»Mit dem Rechenschaftsbericht des Geschäftsführers Dr. Jürgen Bolten beendete damit der Arbeitskreis Deutsch als Fremdsprache $(\mathrm{AkDaF})$ beim DAAD seine Tätigkeit«. (Bolten 1990: 322)

Wie sehr der neue Verband nötig war, zeigt unter anderem, dass sich in den wenigen Monaten zwischen Gründung und der Jahrestagung 1990 in Bonn 150 Mitglieder angemeldet hatten.

\section{Entwicklungslinien}

Die ersten Jahre des neuen Verbandes waren gekennzeichnet durch Öffnungen und neue Horizonte. Die Öffnung war zunächst eine geografische und ging dem damaligen Zeitgeschehen entsprechend nach Osten. Ich erinnere mich gut an aufregende und fruchtbare Diskussionen mit den DDR-KollegInnen. Für diesen überschaubaren Bereich hatten wir das gute Gefühl, dass zusammengekommen war, was zusammengehört. Wir haben aber auch erstaunt festgestellt, dass die KollegInnen dort in vielen Bereichen der DaF-Vermittlung und der Lernorganisation weiter waren als wir im Westen. Im Curriculum war z. B. geregelt, dass ausländische Studierende auch noch in den ersten vier Semestern des Fachstudiums obligatorisch DaF-Kurse besuchen und darüber Prüfungen ablegen mussten. Weiter waren sie auch in der engen Verzahnung zwischen Forschung und Lehre, in den Bereichen Phonetik und in der Erforschung und Vermittlung von Fachsprachen.

Es war aber offensichtlich unmöglich, Anfang der 90er Jahre etwas von dort zu übernehmen, weil aus westdeutscher Sicht die Bewertung aller Erscheinungen der DDR subsumiert wurde unter dem damaligen Lieblingsadjektiv der Wessis: $\gg$ marode $\ll$.

Nebenbei erfuhren wir auch viel über die Befindlichkeiten in der DDR, zum Beispiel, dass in der politischen Klasse offensichtlich genauso gerangelt und hintergangen wurde wie im Westen. So lautete ein bekannter Spruch unter SED-Parteimitgliedern: »Den Helm eines aufrechten Sozialisten zieren viele Dellen - die wenigsten davon stammen vom Klassenfeind.« 
In den Anfangsjahren des Verbands gab es unterschiedliche Vorstellungen darüber, ob er sich weiterhin primär an den Lehrgebieten und Studienkollegs orientieren sollte. Die Alternative war, sich auch für die außeruniversitären, kommerziellen Anbieter zu öffnen. Das letztere ist dann eingetreten und hat in gewisser Weise den großen Boom der privaten Anbieter antizipiert. Allein die Zahl der Anbieter von Integrationskursen beträgt heute ca. 1.500 mit etwa 17.600 Lehrenden und über 150.000 Kursteilnehmern pro Jahr. ${ }^{1}$

Durch diese Öffnung drangen ganz neue und für einige im $\mathrm{FaDaF}$ befremdliche Begriffe wie »Qualitätskriterien «, »Qualitätsstandards«, »Marketing « und später noch »Zertifizierung « in die geschützten Räume der Lehrgebiete und Studienkollegs ein. Aus der Rückschau muss man sagen: »Und das war gut so.«

Es war der Übergang bzw. der Spagat zwischen den Konzepten »Institution « und »Anbieter «, also zwischen Institutionen, die DaF-Unterricht erteilen, und Anbietern von Dienstleistungen im Bereich DaF. Der FaDaF hat im Laufe der Jahre beide Konzepte integriert. So ist es vielleicht nicht verwunderlich, für manche aber sicher überraschend, dass heute die Einnahmen des FaDaF zu einem Drittel im weiteren Sinne aus dem Verkauf von Dienstleistungen kommen.

Der FaDaF begann dann schon sehr früh, sich auf Messen zu präsentieren, zum Teil noch bevor die Universitäten selber sich auf diese Weise um neue Klientel im Ausland bemühten. Der Besuch solcher Bildungsmessen ist heute der Normalfall geworden.
Die Öffnung bezog auch DAAD-LektorInnen und Sprachassistenten mit ein. In den zurückliegenden Jahren hat der FaDaF verstärkt auch den Bereichen Deutsch als Zweitsprache, Integrationskursen und deren Anbietern ein Forum geboten.

Es ist m.E. eine große Leistung des $\mathrm{FaDaF}$, diese unterschiedlichen Gruppen, Interessen und Fachfelder integriert zu haben. Denn für all diese Bereiche könnte es ja auch eigene Organisationen, Verbände oder Arbeitskreise geben.

Und noch ein wichtiges Ereignis dieser ersten Jahre soll erwähnt werden: 1993 wurde der FaDaF nach langen Bemühungen Mitglied des Internationalen Deutschlehrerverbandes (IDV) und hat in der Folge auch verschiedene Verbände aus Entwicklungsländern finanziell unterstützt. $^{2}$

Durch die Jahrestagungen, die vielen Fachtagungen, Workshops, Regionalkonferenzen und andere Fortbildungsveranstaltungen ist ein kontinuierlicher Austausch zwischen Praktikern und der wissenschaftlich-didaktischen Entwicklung des Faches entstanden. Daraus hat sich auch eine z.T. enge Verzahnung zwischen Mitgliedern und Vorstand ergeben. Es herrschte fast immer eine gute Atmosphäre, so dass sozusagen als Kollateralgewinn viele Freundschaften entstanden sind. Und es ist sicher auch zu Verliebungen und nachweislich auch zu Heiraten gekommen. Deshalb können alle Mitglieder, Vorstand und Beirat - zu Recht sagen: »Wir sind der FaDaF.«

Diese Integration gelang auch deshalb, weil - nach meinem Eindruck - diese z. T. sehr spezifischen Interessen und Bedürfnisse nicht nur durch die o. e. vielen und

1 Ich danke Amadeus Hempel für diese Zahlen.

2 Solche Unterstützungsaktionen gab es z.B. für Bulgarien, Kamerun, Mazedonien, Aserbaidschan, Senegal u. a. 
vielfältigen Veranstaltungen, sondern zunehmend auch durch Serviceleistungen des Verbandes (z.B. die Förderung des Nachwuchses, der Stellenpool und die Erarbeitung von Prüfungssets für die zentralen PNdS- bzw. DSH-Prüfungen) weitgehend befriedigt werden konnten. Ich denke, diese Gruppen hatten meist das Gefühl, das man mit dem bekannten Satz einer nicht mehr so bekannten Deutsch-Bolivianerin ausdrücken kann: "Da werden Sie geholfen. «"

Nun sind nicht immer alle mit allem zufrieden. Aber dann sollte das Motto lauten: "Mitmachen statt motzen." Ich habe in den letzten Jahren mehrfach das Amt des Wahlleiters für die Wahl des Vorstands im Rahmen der Mitgliederversammlung übernommen und dabei immer wieder die Formalia erklärt. Aber die Formalia waren nicht das Problem. Das Problem war meist, einen zehnten Kandidaten oder eine zehnte Kandidatin zu finden, der/die die Wahl überhaupt erst notwendig machen würde. Hier könnte das »Mitmachprinzip« noch gestärkt werden.

\section{Mitgliederzahlen}

Ich hatte gesagt, dass sich in den wenigen Monaten zwischen Gründung und erster Jahrestagung in Bonn 150 Mitglieder angemeldet hatten. Heute sind es ca. 800 . Insgesamt gab es aber in diesen 20 Jahren viel mehr Mitglieder. Eine ganze Reihe ist ausgetreten oder verstorben, andere wurden als Karteileichen herausgenommen. Und eine größere Zahl von Mitgliedsnummern ist nicht vergeben worden.

Werner Roggausch, der langjährige Vertreter des DAAD im FaDaF, hat mit sei- nem Ausscheiden aus dem DAAD vom FaDaF-Vorstand als Ehrenmitglied die Mitgliedsnummer 3.333 bekommen. Vielleicht wollte sich der Verband damit unbewusst ein langfristiges, quantitatives Ziel setzen. Auf jeden Fall: Wer heute oder morgen früh Mitglied wird, erhält die Mitgliedsnummer 2.275. Damit wäre der Verband gerade mal knapp 1.000 Mitglieder von der magischen Zahl 3.333 entfernt, die er aufgrund meiner Hochrechnung ca. 2020 erreichen könnte!

\section{Errungenschaften und Leistungen}

Ganz entscheidend für die kontinuierliche Entwicklung des Verbandes war die Einrichtung einer permanenten Geschäftsstelle 1992/93 in Münster ${ }^{2}$. Idee und Durchführung damals: Winfried Welter vom Lehrgebiet der Universität Münster. Das hat so kein anderer Verband im Bereich der Philologien; ist also - wie man heute sagt - ein Alleinstellungsmerkmal.

Viel Energie ist auch in die Entwicklung der Deutschen Sprachprüfung für den Hochschulzugang (DSH) als Nachfolgerin der PNdS geflossen. Das DSH-Handbuch wurde in vielen Konferenzen, Fachtagungen und Workshops diskutiert und erarbeitet, ebenso wie Prüfungssets für zentrale DSH-Prüfungen.

Auf den Jahrestagungen der letzten 20 Jahre wurden ca. 90 Themenschwerpunkte angeboten und im gleichen Zeitraum wurden fast 50 Fachtagungen $\mathrm{zu}$ den unterschiedlichsten Themenbereichen organisiert (siehe dazu den Anhang).

Herausgreifen möchte ich die Leitthemen der Tagung 1991 in Berlin, nämlich »DaF

1 Diesen Satz hat die Fernsehmoderatorin Verona Feldbusch gesagt und mehrfach auch als Werbeslogan verwendet.

2 Die Geschäftsstelle befindet sich gegenwärtig an der Universität Göttingen und ist dort an die Abteilung »Interkulturelle Germanistik« am Seminar für Deutsche Philologie angebunden. 
ohne Mauern « und der diesjährigen Tagung hier in Freiburg: »Grenzen überwinden mit Deutsch «. Sie markieren auch eine Hinwendung vom Blick nach innen, der 1991 verständlich war, zu dem Blick nach außen.

Als besondere Leistung des FaDaF möchte ich noch die »Kommentierte Auswahlbibliographie von Neuerscheinungen für das Fach Deutsch als Fremdsprache « herausstellen, die einmal pro Jahr in Info DaF erscheint. Diese hat Dietrich Eggers mit den Mainzer MitarbeiterInnen schon in der AkDaF-Zeit auf den Weg gebracht und auch weit in die FaDaF-Zeit hinein dann mit Evelyn Müller-Küppers und Lutz Köster - weiter betreut. Dort erscheinen jährlich ca. 100 Rezensionen (!), also ca. 2.000 in den letzten 20 Jahren, und zwar jeweils ca. ein Jahr nach Erscheinen der besprochenen Bücher. Das ist - bescheiden ausgedrückt - sensationell. Das leistet kein anderer vergleichbarer Verband.

Diese Leistungen wären nicht oder nicht so möglich gewesen ohne die kontinuierliche finanzielle Unterstützung durch den DAAD. Der verantwortliche Mittler zwischen den zwei Organisationen war von 1990 bis 2008 Werner Roggausch. Er hat die meisten Wünsche und Interessen des FaDaF mitgetragen, manchmal auch in konstruktiv-kritischer Solidarität. Wir sollten dem DAAD und Dr. Werner Roggausch und seiner Nachfolgerin, Frau Dr. Schneider, für diese großartige Unterstützung danken.

Diese Leistungen wären aber auch nicht möglich gewesen ohne das immense Engagement der jeweiligen Vorstandsmitglieder und weiterer Mitglieder, die Aufgaben übernommen haben, z. B. Schwerpunkte der Jahrestagungen zu organisieren, Fachtagungen durchzuführen, Materialien zu erstellen, im Beirat des Verbandes und im Redaktionsbeirat von Info DaF mitzuarbeiten.
Die Belastungen im Vorstand und vor allem der jeweiligen Vorsitzenden gingen oft an die Grenzen der physischen Leistungsfähigkeit. Viele auch außerhalb des Vorstands und in der Geschäftsstelle haben sich ebenfalls mit großer Kraft für den $\mathrm{FaDaF}$ eingesetzt. Ich möchte explizit auch Dietrich Eggers mit einschließen, der sozusagen die Leitfigur des AkDaF war und den $\mathrm{FaDaF}$ mit auf den Weg gebracht hat. Stellvertretend für alle, an die unser Dank gehen muss, nenne ich noch einmal die Vorsitzenden der letzten 20 Jahre: Jürgen Bolten (1989-1993), Armin Wolff (1993-1995), Bernd Wintermann (1995-2001), Hiltraud CasperHehne (2001-2009) und Matthias Jung (ab 2009).

\section{Öffentlichkeitsrelevanz}

Die Öffentlichkeitsrelevanz und damit vielleicht auch die Attraktivität des FaDaF kann man am leichtesten im Vergleich mit anderen Verbänden ermitteln. Ich habe deshalb die relevanten Verbände in eine Suchmaschine eingegeben und sie dann nach der Zahl der Einträge angeordnet.

Ergebnis der Google-Suche am 8.5.2010:

\begin{tabular}{|l|c|}
\hline $\begin{array}{l}\text { Fachverband Deutsch als Fremd- } \\
\text { sprache }\end{array}$ & 93.400 \\
\hline Germanistenverband & 23.500 \\
\hline $\begin{array}{l}\text { Gesellschaft für angewandte Lin- } \\
\text { guistik }\end{array}$ & 23.200 \\
\hline Romanistenverband & 22.300 \\
\hline $\begin{array}{l}\text { Gesellschaft für interkulturelle } \\
\text { Germanistik }\end{array}$ & 17.500 \\
\hline Anglistenverband & 3.850 \\
\hline
\end{tabular}

Auch wenn der FaDaF auf der letzten Stelle gelandet wäre, hätte ich diese Übersicht gezeigt. Aber so können wir dieses Ergebnis als schönes Geburtstagsgeschenk für den $\mathrm{FaDaF}$ ansehen. 


\section{Anregungen}

Trotz des positiven Bildes, das ich gezeichnet habe, möchte ich dem $\mathrm{FaDaF}$ ein paar Anregungen mit auf den Weg geben:

1. Der Kontakt zu Verbänden anderer Philologien könnte m. E. verstärkt werden, und sei es auch nur, um thematisch oder organisatorisch voneinander zu lernen oder in Projekten zu kooperieren. In diesem Zusammenhang hat Werner Roggausch öfter darauf hingewiesen, dass die Sprachdozenten in ausländischen Germanistenverbänden eine interessante Klientel wären, um die sich der $\mathrm{FaDaF}$ stärker kümmern sollte. Der FaDaF hat ja schon Mitglieder aus mindestens 26 nichtdeutschsprachigen Ländern. Einige weiter entfernte sind: Iran, Hong Kong, Taiwan, Thailand, Australien, Südkorea, USA und Kanada. Darauf könnte man aufbauen.

2. Der FaDaF sollte auch mehr auf die so genannten »digital natives « zugehen im Sportjargon wäre das die U 30, also die Generation, die mit dem Internet groß geworden ist. Das sind die, denen beim Wort "Netz « überhaupt nicht mehr einfällt, dass das etwas mit Fischerei zu tun haben könnte.

3. Zur Reihe "Materialien Deutsch als Fremdsprache«: Die jetzige Regelung mit dem Verlag besagt, dass die MatDaF-Bände zwei Jahre nach Erscheinen ins Netz gestellt werden. Ich meine, man sollte zumindest die Vorträge der Jahrestagungen sobald wie möglich nach der Tagung ins Netz stellen. Das käme vor allem denen zugute, die die Tagung nicht besuchen konnten und damit auch vielen DaFlern im Ausland.
Aber auch die Tagungsteilnehmer könnten nachträglich viel mehr aus dem Tagungsangebot herausholen als durch den Besuch von 10-15 Vorträgen. Dieses Angebot könnte interaktiv gestaltet werden, so dass die Nutzer mit den AutorInnen auch nach der Tagung diskutieren könnten.

4. Auf der Homepage des FaDaF wurde vor einigen Jahren die »Mailingliste Forum DaF « eingerichtet, die offensichtlich leise vor sich hindümpelt. Zur Einführung heißt es dort: »Die DaFMailingliste dient dem Austausch und der Diskussion von Themen aus dem Fachgebiet Deutsch als Fremdsprache. Sie ist für jeden Interessenten offen." Man sollte sie bekannter machen und mit Hilfe einer guten Moderation neu beleben und damit den Austausch anregen.

5. Als letztes möchte ich anregen, dass die Verantwortlichen für die DSH und für den TestDaF aufeinander zugehen, um zu Annäherungen oder gar einer Vereinheitlichung zu kommen.

Der FaDaF muss sich nicht neu erfinden. Aber solche und weitere gezielte Verbesserungen könnten so etwas wie einen FaDaF 2.0 auf den Weg bringen.

\section{Schlussbemerkung}

So, ich bin wohl schon in der Nachspielzeit ...

Ich habe anfangs gesagt, dass dies keine Festrede werden würde. Vielleicht haben einige fälschlicherweise befürchtet, dass es eine Abrechnung werden könnte. Wenn es eine solche geworden wäre, könnte ich jetzt einen anderen Giganten der deutschen Sprache zitieren und sagen: »Ich habe fertig. «1

1 Das ist ein berühmt gewordener Satz des ehemaligen italienischen Trainers von Bayern München, Giovanni Trappatoni, am Ende einer Schimpftirade über einige Spieler des Vereins. 
Ich könnte aber auch sagen, um doch noch einen Hauch von Festreden-Flair hineinzubringen: "Ad multos annos." Aber das finde ich für einen 20-jährigen Spund doch etwas zu sperrig. Ohnehin wäre das ja nur die Aufforderung, etwas zu tun, was man ohnehin nicht vermeiden kann: nämlich älter werden. Im Gegenteil, ich meine, das Motto sollte lauten: jung bleiben. Das könnte man hier sogar als Wortspiel verstehen.

Nur wer bereit zu Aufbruch ist und Reise, Mag lähmender Gewöhnung sich entraffen. (Hermann Hesse: Stufen)

Meine Frau und ich stehen jeden Morgen um Viertel nach sieben auf der Matte im Wohnzimmer und machen Gymnastik mit dem Bayerischen Fernsehen. Von einer der Vorturnerinnen werden wir dann nach 15 Minuten jedes Mal mit der Aufforderung verabschiedet: »Bleiben Sie in Bewegung.« Ich möchte das über das Körperliche hinaus verstehen und im Sinne von »Wir sind der $\mathrm{FaDaF}$ « sagen: »Bleiben wir in Bewegung!«

\section{Literatur}

Bolten, Jürgen: »Fachverband Deutsch als Fremdsprache«. Ein Bericht, Info DaF 17 (1990), 322.

Hesse, Hermann: Die Gedichte. Frankfurt a. M.: Suhrkamp, 1977 (stb 381).

Schulte, Hans-Gerd: »Einführung des Deutschen Akademischen Austauschdienstes zur 1 . Ausgabe von Info DaF «, Info DaF 1 (1974), 1.

Weinrich, Harald: »Deutsch als Fremdsprache - Konturen eines neuen Faches«. In: Wolff, Armin; Jauß, Kurt-Werner (Hrsg.): Didaktische und methodische Beiträge Deutsch als Fremdsprache. Beiträge der Jahrestagung DaF 1978. Regensburg: AkDaF, 1979, 15-35 (Materialien Deutsch als Fremdsprache, 14).

Wolff, Armin: «5 Jahre Arbeitskreis Deutsch als Fremdsprache beim DAAD «. In: Wolff, Armin; Jauß, Kurt-Werner (Hrsg.): Didaktische und methodische Beiträge
Deutsch als Fremdsprache. Beiträge der Jahrestagung DaF 1978. Regensburg: AkDaF, 1979, 12-14 (Materialien Deutsch als Fremdsprache, 14).

Wolff, Armin; Eggers, Dietrich; Ehnert, Rolf; Kirsch, Klaus: »Deutsch als Fremdsprache und der Studienstandort Deutschland. Entwicklungslinien des Faches aus der Sicht (s)eines Verbandes", Info DaF 5 (1997), 559-586.

\section{Anhang}

(Diese Anhänge hat Armin Wolff zusammengestellt. Bei den Jahrestagungen sind die Leitthemen recte und die Themenschwerpunkte kursiv gesetzt.)

\section{Jahrestagungen des Fachverbandes Deutsch als Fremdsprache (FaDaF)}

1990 in Bonn: Deutsch als Fremdsprache im europäischen Binnenmarkt. Deutsch als Fremdsprache im europäischen Binnenmarkt - Grammatik und Grammatikvermittlung Fiktionale Texte im Sprachunterricht - Computer und Computerunterstützung im Bereich Deutsch als Fremdsprache - Forum Deutsch als Fremdsprache.

1991 in Berlin: DaF ohne Mauern. Prüfen und Testen - Wortschatzarbeit - Landeskunde neu - Deutsch als Fremdsprache im Ausland.

1992 in Münster: Mündliche Kommunikation - Unterrichts- und Übungsformen DaF Themen- und Zielgruppenspezifische Auswahl von Unterrichtsmaterialien - Modelle für studien- und berufsbegleitenden Unterricht.

1993 in Erlangen: Autonomes Lernen - Lernpsychologie im Fremdsprachenunterricht Deutsch als Fremdsprache im internationalen Kontakt - Qualitätskriterien für Sprachkurse DaF im außeruniversitären Bereich.

1994 in Aachen: Fach- und Sprachunterricht: Gemeinsamkeiten und Unterschiede. Studienkollegs Deutsch als Fremdsprache. Von der Theorie zur Praxis. Fach- und Sprachunterricht: Gemeinsamkeiten, Unterschiede und Kooperationsmöglichkeiten - Zur mündlichen Kommunikation in Vorstudienkursen - Fach- und Berufssprache: Angebot und Nachfrage - Studiengänge Deutsch als Fremdsprache: Von der Theorie zur Praxis - Traditionelle Lernmethoden: Neue Möglichkeiten. 
1995 in Dresden: DaF für die Zukunft. Eine Zukunft für DaF! Ausländerstudium in einem sich verändernden Deutschland Multimedia und Fremdsprachenlernen Üben und Prüfen in Fach- und Berufssprachen - Landeskunde und kulturelle Kompetenz.

1996 in Göttingen: Gedächtnis und Sprachlernen - Prozeßorientiertes Fremdsprachenlernen-Deutschlehrerausbildung in West-und Osteuropa - Eine deutsche Literatur: AutorInnen nichtdeutscher Muttersprache.

1997 in Mainz: Emotion und Kognition beim Fremdsprachenlernen - Lernen mit neuen Medien - Deutsch als Fremdsprache und die Attraktivität des Studien- und Lernortes Deutschland - Forum Deutsch als Fremdsprache.

1998 in Jena: Alternative Vermittlungsmethoden und Lernformen auf dem Praxisprüfstand - Wissenschaftssprache, Fachsprache Landeskunde aktuell - Interkulturelle Begegnung, Interkulturelles Lernen.

1999 in Regensburg: Sprache - Kultur Politik. Die Stellung des Deutschen als Fremdsprache im Rahmen der KulturpolitikEmpirische Forschung und ihre Auswirkungen auf die Praxis - Testen und PrüfenQualitätssicherung.

2000 in Dortmund: Wie schwer ist die deutsche Sprache wirklich? Deutsch als Fremdsprache, Deutsch als Zweitsprache, Deutsch als Muttersprache - Alltägliche Wissenschaftssprache und Sprachkurse in der $\mathrm{Zu}$ kunft: studienvorbereitend und studienintegriert - Deutsch für den Beruf-Wie schwer ist Deutsch wirklich?

2001 in Kiel: Europäisches Jahr der Sprachen: Mehrsprachigkeit in Europa. Sprachenpolitik und europäische Mobilität - Lernpsychologie und vermittlungsmethodische Aspekte der Mehrsprachigkeit - Anforderungsprofile in der DaF-/DaZ-/DaM-Ausbildung und Berufsqualifikation - Nachweis von Sprachkenntnissen im europäischen Verbund.

2002 in München: Qualifizierungen für eine transnationale Kommunikation. Die Vielperspektivität in Literatur und Literaturvermittlung - Neue Methoden der Grammatikvermittlung - Einheit von Fach- und DaFUnterricht - Integration von Online-Lernen mit neuen Medien.

2003 in Essen: Integration durch Sprache. Sprachförderung in Deutschland: quo vadis?
- Deutsch als Zweitsprache - Deutsch als fremde Wissenschaftssprache studienbegleitend - Testen und Prüfen.

2004 in Bielefeld: Sprache lehren - Sprache lernen. Neuere Entwicklungen in der Sprachlehr-l-lernforschung - Kulturspezifische Wissenschafts- und Unterrichtsstile Mündliche Textproduktion sowie -rezeption und ihre Vermittlung - Standards - Qualität - Politik.

2005 in Jena: Umbrüche. Sprache und Gehirn - Landeskunde/Cultural Studies - Deutsch als Fremdsprache: Materialien und Medien Die Ökonomisierung der Bildung und Deutsch als Fremdsprache.

2006 in Hannover: Die Welt zu Gast bei Freunden. Integrationskurs DaF - Testen und Prüfen, Korrektur und Bewertungsverfahren - Sprechen und Schreiben in den Wissenschaften - Deutsch als Zweitsprache und Mehrsprachigkeit.

2007 in Berlin: Auf neuen Wegen: Deutsch als Fremdsprache in Forschung und Lehre. Empirische Forschung im Bereich Deutsch als Fremdsprache - Grammatik: Theorie und Praxis für den DaF-UnterrichtFachsprache und ihre Vermittlung - Gesprochene Sprache.

2008 in Düsseldorf: DaF integriert: literatur: medien: ausbildung. Literatur, Theater und Film - Mediengestütztes Lernen - Lehreraus-und-fortbildung - Integrationskurse.

2009 in Jena/Weimar im Rahmen der IDT nur eine Mitgliederversammlung.

2010 in Freiburg: Grenzen überwinden mit Deutsch. Mehrsprachigkeit in Grenzregionen - Kompetenzen beschreiben, fördern, evaluieren - Motivation: Forschungsgegenstand und Unterrichtspraxis - Wirkung von Unterricht auf das Lernen von Sprachen.

\section{Fachtagungen des Fachverbandes Deutsch als Fremdsprache}

1990 in Regensburg: Phonetik, Ausspracheschulung, Sprecherziehung im Bereich Deutsch als Fremdsprache (in Zusammenarbeit mit der Deutschen Gesellschaft für Sprecherziehung und Sprechwissenschaft).

1990 in Bonn: Workshop im Rahmen der GAL: Deutsch als Fremdsprache in den neunziger Jahren.

1991 in Göttingen: Schreiben.

1991 in Leipzig: Studienbegleitende Deutschkurse. 
1991 in Mainz: Workshop im Rahmen der GAL: Zu einigen Perspektiven des Faches DaF für die neunziger Jahre.

1992 in Regensburg: Reform der PNdS-Prüfung.

1992 in Chemnitz: Deutschsprachige Fachkommunikation im universitären und außeruniversitären Bereich.

1993 in Leipzig: DaF-Unterricht im Fachunterricht.

1995 in Jena: Medieneinsatz im Fremdsprachenunterricht unter besonderer Berücksichtigung von Multimedia.

1996 in Regensburg: Zur DSH I: Umsetzung der neuen DSH-Ordnung in die Prüfungspraxis.

1997 in Regensburg: Zur DSH II: Umsetzung der neuen DSH-Ordnung in die Prüfungspraxis.

1997 in Leipzig: Symposium der Studiengänge Deutsch als Fremdsprache.

1998 in Münster: Studienintegrierte Sprachvermittlung DaF an Hochschulen - Realisierung, Bewertung und Zertifizierung.

1998 in Bad Emstal: DSH: Bewertungskriterien und Korrekturverfahren.

1998 in Chemnitz: IDV-Fachsprachensymposion im Auftrag des FaDaF: Fachkommunikation 2000. Kompetenzprofile für Lehrende an universitären und außeruniversitären Einrichtungen des In- und Auslandes.

1998 in Potsdam: Zusammen mit dem Studienkolleg Potsdam: Fachtagung Deutsch als Fremdsprache für Lehrkräfte an Studienkollegs.

1999 in Bielefeld: Symposium der Studiengänge zu den Praktika in der DaF-LehrerAusbildung.

1999 in Braunschweig: Studienbegleitende und studienintegrierte Sprachkurse Deutsch als Fremdsprache an Hochschulen.

1999 in Frankfurt: Workshop im Rahmen der GAL: Referenzrahmen und die zugrunde liegenden Sprachbeschreibungs- und -aneignungsmodelle.

2000 in Blaubeuren: Phonetik und mündliche Kommunikation.

2001 in München: Workshop: Sprachenlernen und-lehren im Internet.

2001 in Düsseldorf: Internet im DaF-Unterricht.

2001 in Rauischholzhausen: Zur Rolle der Praktika in den DaF-Studiengängen.
2002 in Blaubeuren: Phonetik und interkulturelle Kommunikation.

2002 in Braunschweig: Studiumsbegleitender Sprachunterricht - Hilfen zur Lernbeschleunigung.

2002 in Braunschweig: DSH und TestDaF: Politische Implikationen und wissenschaftliche Erforschung. Initiierung einer Vergleichsstudie.

2002 in Bonn (gemeinsam mit dem DAAD und den Studienkollegs): Fachtagung im Rahmen des Bologna-Prozesses: »Education for Transition «.

2003 in Göttingen: DSH: Neue Rahmenordnung und neues Handbuch. Auf dem Weg zu einer weiteren Standardisierung. Zur Entwicklung einer neuen Rahmenordnung für TestDaF und DSH.

2003 in Oldenburg: Forschung ist international -Deutschkursangebot spielt eine zentrale Rolle bei der Integration ausländischer Studierender und WissenschaftlerInnen in Universität und Gesellschaft.

2004 in Jena (zusammen mit Sokrates/ Grundtvig-Projekt CHAGAL): Internationale Studierende auf ihrem Weg in die Hochschulen. Neue Ansätze für studienvorbereitende Sprach- und Orientierungsangebote.

2004 in Blaubeuren (zusammen mit dem FMF): Phonetik und Kommunikation.

2004 in Göttingen: Workshop zur Neuerstellung des DSH-Handbuchs.

2005 in Düsseldorf: Workshop: Universitätsnah wirtschaften mit DaF.

2005 in Bochum: Lernberatung.

2005 in Hannover: Bachelor und Master in Deutsch als Fremdsprache: Probleme und Perspektiven.

2006 in Hannover: Workshop: Chancen für den DaF-/DaZ-Nachwuchs.

2006 in Jena DSH: Deutsche Sprachprüfung für den Hochschulzugang ausländischer Studienbewerberinnen und Studienbewerber.

2007 in Leipzig: Quo vadis DaF-Nachwuchs: Forschungsthemen und -designs.

2007 in Berlin: Integrationskurse.

2007 in Mannheim: Frühjahrstreffen des Arbeitskreises »Wirtschaften mit DaF«.

2007 in Göttingen: Sprachpraxis der DaFund Germanistikstudiengänge im europäischen Hochschulraum.

2007 in Essen: Deutschsprachige Studiengänge und bilinguales Lehren und Lernen. 
2007 in Düsseldorf: Sprachliche Frühförderung für Kinder mit Migrationshintergrund.

2008 in Blaubeuren (in Zusammenarbeit mit dem GMF, Sektion DaF): Wege zum freien Sprechen.

2008 in Paderborn: Frühjahrstreffen des FaDaF-Arbeitskreises »Wirtschaften mit $D a F \ll$.

2009 in Oldenburg: Forschungsmethoden im Fach Deutsch als Zweit- und Fremdsprache.

2009 in Augsburg: Sprachliche Förderung von Kindern mit Migrationshintergrund.

2009 in Bielefeld: Studienbegleitende Angebote zum akademischen Schreiben in der Fremdsprache Deutsch.

2009 in Essen: Deutsch als Zweitsprache in der Lehrerausbildung.

2010 in Freiburg: Tagung des wissenschaftlichen DaF-Nachwuchses.
Klaus Vorderwülbecke

Studium der Anglistik, Geschichte und Pädagogik an den Universitäten Bonn, Aix-en-Provence, Wien und Washington State University, USA; nach dem Studium vier Jahre DAAD-Lektor an der Hokkaido-Universität in Sapporo, Japan. Von 1973 bis 2005 wissenschaftlicher Mitarbeiter am Institut für Deutsche Sprache, Mannheim; 1993-97 beurlaubt für die Mitarbeit an dem Fernsehsprachkurs »Einblicke« von Goethe-Institut und Inter Nationes; Mitautor mehrerer DaF-Lehr-Lernmaterialien; Fortbildungsseminare für DaF-Lehrende im In- und Ausland; thematische DaF-Schwerpunkte: Lehrwerkanalyse, Phonetik, Landeskunde. 